\title{
RENACER DESPUÉS DE LA TRAGEDIA: UNA MIRADA BREVE A LA RELACIÓN ENTRE DEMOGRAFÍA Y CATÁSTROFE EN CHILE*
}

Enrique Aliste y Rodrigo Moreno

* Este texto se ha podido realizar gracias al proyecto FONDECYT 1090248. 


\section{ENRIQUE ALISTE}

Geógrafo. Magíster en Gestión y Planificación Ambiental, Universidad de Chile. Es actualmente candidato a Doctor en Estudios Comparativos sobre el Desarrollo en l'École des Hautes Études en Sciences Sociales (EHESS) de París, Francia. Profesor del Departamento de Geografía de la Facultad de Arquitectura y Urbanismo y del Departamento de Ciencias Históricas de la Facultad de Filosofía y Humanidades de la Universidad de Chile. Investigador Responsable y co-investigador de proyectos Fondecyt. Ha sido profesor invitado en la Universidad de Poitiers y en el Institut des Hautes Études de l'Amérique Latine (IHEAL-CREDAL) de la Universidad de Paris III, Francia.

\section{RODRIGO MORENO}

Geógrafo. Diploma en Investigación Social en Integración de los Migrantes Internacionales, y candidato a Magíster en Geografía con mención en Ordenamiento Territorial, Urbano-Regional, Universidad de Chile. Actualmente se desempeña como académico del Departamento de Geografía de esta Casa de Estudios. Ha participado en diversos proyectos de investigación Fondecyt. 


\section{INTRODUCCIÓN}

En el año 1948, el destacado historiador francés Fernand Braudel, publicó en la revista Anales: Economías, Sociedades, Civilizaciones el artículo "Chile, aquella locura geográfica". Destacaba en este artículo el hecho de que se trataba de "el país más trágico de América. Ello quiere decir, sobretodo, el más humano..."2. ¿Qué quiere decirnos Braudel en esta aseveración? Se trata de un comentario crítico al trabajo de Benjamín Subercaseaux Chile, o una loca geografía ${ }^{3}$, en donde además de verter sus alusiones al texto del escritor y médico chileno, lo hace también en torno a las características geográficas y físicas de nuestro territorio. Muchas son sus expresiones relativas a las particulares condiciones geográficas de Chile, en donde frases como "uno de los países más curiosos de América Latina", o "que cada sufrimiento obliga al hombre a reaccionar y readaptarse" van poco a poco dibujando y dejando ver un imaginario en torno a una tierra indómita, salvaje, y sobretodo muy propensa a las adversidades que la naturaleza ofrece a la ocupación humana.

Uno de los temas que se han observado en relación con estas formas de reacción y readaptación dice relación con las respuestas en torno al comportamiento demográfico post catástrofe. Numerosos artículos de prensa destacan esta situación, la que debe mirarse con cautela: más elementos para mirar el fenómeno permiten un análisis en perspectiva.

Por lo pronto, la prensa escrita desde mediados del siglo XX ya acusaba situaciones de naturaleza similar en diferentes catástrofes no solo nacionales sino también internacionales. Para ello, se utiliza por lo general el anglicismo baby boom, que se acuña luego del periodo de incremento de la natalidad ocurrido al finalizar la Segunda Guerra Mundial, particularmente en Estados Unidos,

1. Braudel, F. "Chili, cette folie géographique". En: Annales. Économies, Sociétés, Civilisations. $3^{e}$ année, No4, 1948, pp. 443-446.

2. Op. Cit., p. 445.

3. Subercaseaux, B. Chile o una loca geografía. Ediciones Ercilla, 1946, p. 427. 
Australia y Canadá, al regresar a sus respectivos países el contingente de jóvenes sobrevivientes al conflicto ${ }^{4}$.

En Chile, su asociación se da más bien por el vínculo a las catástrofes de causa natural, y el caso de los terremotos y maremotos son probablemente los que despiertan mayor curiosidad en torno al fenómeno. Durante el año 2010, artículos aparecidos en la prensa escrita chilena dan cuenta de esta situación en crónicas que destacan epígrafes como "(...) a días de cumplirse 9 meses del terremoto del 27 de febrero, las ciudades más afectadas por el sismo presentan un considerable aumento de los embarazos"s. La opinión de sociólogos, psicólogos y otros expertos en el área de las ciencias sociales, señalan que el fenómeno puede estar relacionado con un reordenamiento de los aspectos valóricos, instinto de supervivencia, mayor permisividad, entre otras razones. Y situaciones de naturaleza similar se han evidenciado en catástrofes como el terremoto 7.7 grados de la escala Richter registrado en Iquique y Tocopilla en el Norte Grande de Chile en noviembre del año 2007, en donde el número de nacimientos se habría triplicado con posterioridad a la catástrofe.

Frente a la inquietud, y ante la evidencia -sobretodo del Ministerio de Salud en torno al aumento de las consultas de obstetricia en las zonas afectadas por catástrofes como los terremotos-, una mirada al comportamiento demográfico permite mirar con mayor atención el fenómeno. Y algunos datos disponibles a la fecha (aunque no todos, evidentemente), permiten ensayar algunas reflexiones en torno a lo sucedido frente a otras catástrofes relevantes en el país.

\section{NUESTRA CONDICIÓN TELÚRICA Y LA RESPUESTA HUMANA}

De acuerdo con la información del Servicio Sismológico del Departamento de Geofísica de la Universidad de Chile, desde inicios del siglo XX y hasta la fecha se han registrado en Chile 75 sismos cuya magnitud está sobre los 7.0 grados y que por lo mismo pueden ser catalogados como terremotos. Emblemáticos entre ellos son los sismos de 1906 (Valparaíso), 1939 (Chillán), 1960 (Valdivia), 1985 (San Antonio), 2007 (Iquique-Tocopilla) y por cierto, el de 2010 (Cobquecura-Concepción).

Diversas situaciones pueden ser miradas con atención desde el punto de vista de la respuesta demográfica a estas catástrofes y diversas son las condiciones que

4. Russell, C. The baby boom: Americans born 1946 to 1964. New Strategist Publications, 2009, p. 308.

5. El Mercurio. "Alza de embarazos en la zona afectada desata un 'baby boom' en los próximos meses”. Santiago de Chile, edición del sábado 20/11/10, cuerpo C, p. 10.

6. Universidad de Chile. Departamento de Geofísica. Servicio Sismológico. "Sismos importantes y/o destructivos (1570 - a la fecha). Magnitud Ms igual o mayor a 7.0". Documento on line: <http: //ssn.dgf.uchile.cl/seismo.html>. Fecha de consulta: 8/03/11. 
inciden en los balances posteriores. En algunos casos, sin duda que llama la atención el tema de los nacimientos o baby boom. Pero las estadísticas demográficas post catástrofes no pueden por sí solas explicar o ser el reflejo fiel de lo sucedido en torno a este fenómeno. El número de nacimientos es sin duda el dato más relevante que puede obtenerse de los registros que maneja el Ministerio de Salud. Pero en lo relativo a las estadísticas demográficas, bien merece mirar con atención otras fuentes que pueden ayudar a moderar las interpretaciones en tal sentido.

En la línea de lo señalado en el párrafo anterior, los registros de prensa pueden ser pistas de interés. Por ejemplo, el diario El Mercurio en su edición del día 27 de enero de 1939, titulaba en primera plana "Chillán, Cauquenes, Parral, San Carlos y Linares serán evacuadas" lunes 30 de enero de 1939, en el segundo cuerpo del mismo diario, se destaca entre las noticias anunciadas "Convoyes especiales condujeron a Santiago heridos y refugiados". El día 7 de febrero de 1939, el diario El Sur de Concepción destacaba que "6.026 personas evacuaron Concepción controladas por la Gobernación Marítima de Talcahuano". Aquel mismo día, otra noticia señala "200.000 nacionales destinó Argentina como ayuda a damnificados del terremoto"10. En síntesis, se deja ver que en términos de las estadísticas demográficas una importante cantidad de población se desplaza con posterioridad al evento pudiendo afectar la mirada y análisis sobre el proceso.

No obstante lo anterior, y tal como se ve en las estadísticas que se exponen más adelante, hay un notable quiebre de la tendencia demográfica en la ciudad de Chillán, entre 1939 y 1952.

Un aspecto que llama la atención también en torno a la respuesta humana a las condiciones naturales del territorio es la notable disminución en las cifras de víctimas fatales. De los casi 40 mil muertos del terremoto de Chillán en 1939, no se alcanza a llegar a los 3 mil en Valdivia ${ }^{11}$ y poco más de 500 son los oficialmente declarados por el Ministerio del Interior para el reciente sismo del 27 de febrero de $2010^{12}$.

7. El Mercurio. "Chillán, Cauquenes, Parral, San Carlos y Linares serán evacuadas". Santiago de Chile, edición del 27/01/39.

8. El Mercurio. "Convoyes especiales condujeron a Santiago heridos y refugiados". Santiago de Chile, edición del 30/01/39.

9. El Sur. "6.026 personas evacuaron Concepción controladas por la Gobernación Marítima de Talcahuano". Concepción, edición del 7/02/39.

10. El Sur. "200.000 nacionales destino Argentina como ayuda a damnificados del terremoto". Concepción, edición del 7/02/39.

11. <www.sismo24.cl>. Listado de terremotos por ciudades. Fecha de consulta: 10/03/11.

12. Ministerio del Interior. Balance de Reconstrucción a un año del 27/F. Documento on line: <http: //issuu.com/minterior/docs/divest_10207_estudios_especiales_balance_a_1_a_o_d?mode=a_p Ewmode=1>. Fecha de consulta: 7/03/11. 


\section{ALGUNAS CIFRAS SOBRE CATÁSTROFE Y SU CORRELATO DEMOGRÁFICO}

Estadísticas demográficas recopiladas para periodos asociados a algunos eventos catastróficos registrados en el siglo XX, muestran interesantes situaciones que procederemos a mirar con mayor detención. Como muestra se han considerado los eventos catastróficos de los años 1939 (Chillán), 1960 (Valdivia), 1985 (San Antonio) y 2007 (Tocopilla).

Visualmente, los procesos demográficos de localidades afectadas por cada uno de estos eventos son las que se pueden apreciar en los siguientes gráficos:

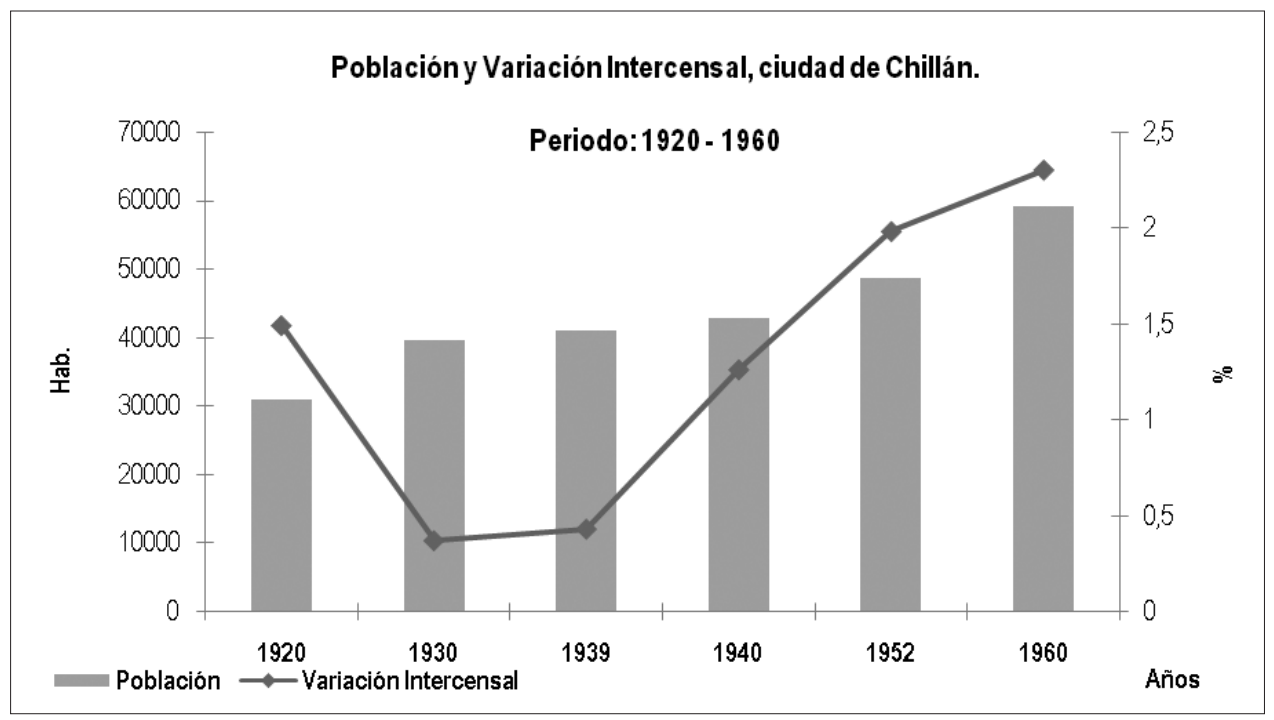

Fuente: INE. Población de los centros poblados de Chile 1875-1992. Santiago de Chile, 1992; INE. Chile: Ciudades, Pueblos, Aldeas y Caseríos. Santiago de Chile, 2005.

Como se puede observar en la gráfica, la ciudad de Chillán venía experimentando un crecimiento sostenido de la población para el periodo 1920-1930, pasando de los 30.000 habitantes a casi los 40.000 , con una variación intercensal del $2,4 \%$, tendencia que debería haberse mantenido y acentuado en el tiempo; no obstante, en la década del 30 se generó un considerable retroceso en este comportamiento, al que habría que agregar los efectos del terremoto de 1939 cuyo balance habla de alrededor de 37.000 víctimas fatales, de los cuales más de un 40\% eran de la ciudad de Chillán ${ }^{13}$. A este escenario se suma la migración de población tanto

13. <www.sismo24.cl>. Documento on line: "24 enero 1939 terremoto Chillán". Fecha de consulta: 9/03/11. 
interna (a ciudades como Concepción desde donde fueron posteriormente evacuados a ciudades como Santiago y Valparaíso principalmente), como al exterior (muchos damnificados emigraron a ciudades de Argentina), producto del miedo a réplicas del evento como a la pérdida de sus viviendas ${ }^{14}$. Lo interesante es evidenciar que, pese al elevado número de víctimas fatales y al fuerte proceso migratorio derivado del alto número de damnificados, la tendencia demográfica se quiebra y tiende al aumento, permitiendo sostener como hipótesis un incremento en el número de nacimientos. Otra hipótesis posible, es sostener que producto del proceso de reconstrucción, pudo haber llegado más gente a la zona, así como procesos migratorios internos desde poblados de menor jerarquía en la región, que hayan llegado a la ciudad de Chillán. La tendencia a partir de la década del 40 en adelante es el crecimiento sostenido de la población, siendo el quiebre más notable entre los años 20 y 60, el experimentado precisamente post catástrofe ${ }^{15}$.

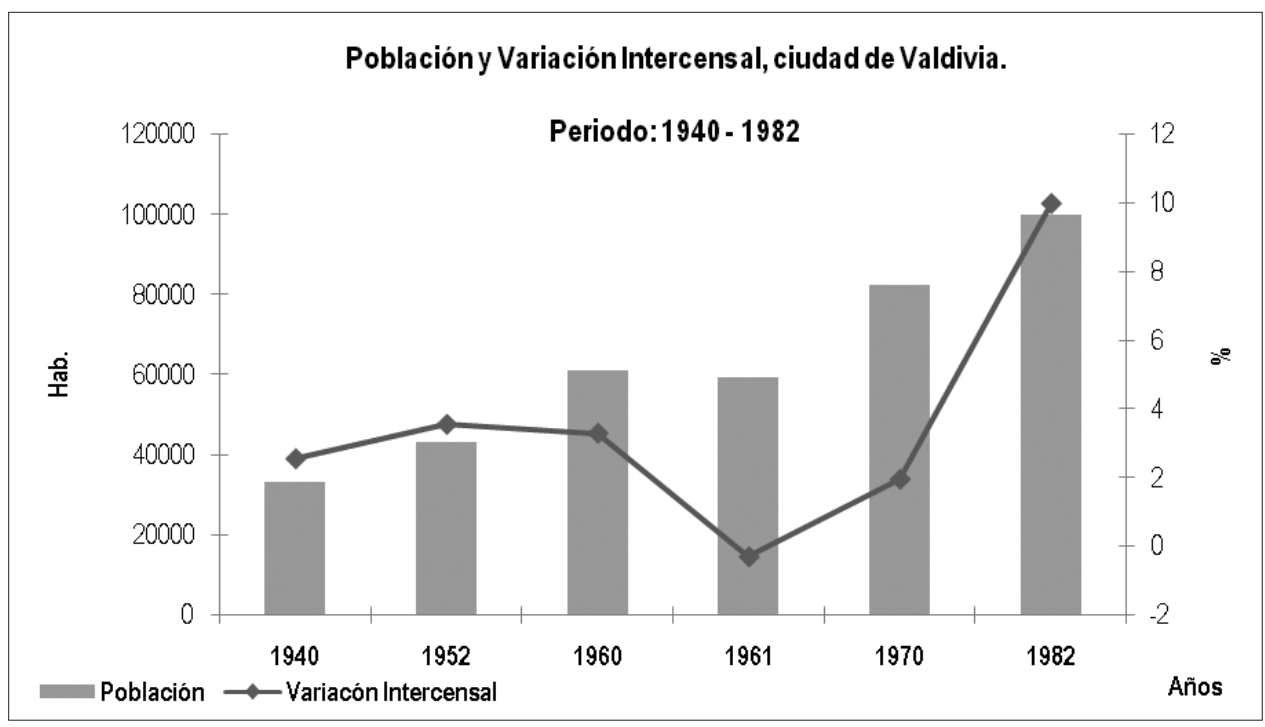

Fuente: INE. Población de los centros poblados de Chile. 1875-1992. Santiago de Chile, 1992; INE. Chile: Ciudades, Pueblos, Aldeas y Caseríos. Santiago de Chile, 2005.

14. El Mercurio. "Convoyes especiales condujeron a Santiago heridos y refugiados". Santiago de Chile, edición del 30/01/39, Segundo Cuerpo, p. 9; El Sur. "6.026 personas evacuaron Concepción controladas por la Gobernación Marítima de Talcahuano. Concepción (Chile), edición del 7/02/39; El Sur. "200.000 nacionales destino Argentina como ayuda a damnificados del terremoto". Concepción (Chile), edición del 7/02/39.

15. Instituto Nacional de Estadísticas, INE. Población de los centros poblados de Chile. 1875-1992. Santiago de Chile, 1992. 
La ciudad de Valdivia, en tanto, y al igual que otras ciudades del sistema urbano chileno para la época, venía presentando un crecimiento económico sostenido ${ }^{16}$, lo que se veía reflejado en su dinámica demográfica presentando una población cercana a los 62.000 habitantes $^{17}$. Pero como se ve en la gráfica, este comportamiento tiene un brusco cambio que indudablemente se asocia al terremoto que azotó a la ciudad en el año 1960, donde se pasó de una variación intercensal positiva del $3.5 \%$ (que era la tendencia), a una del $-0.3 \%$, contabilizándose una disminución en la población cercana al 10\% (asociada principalmente a migraciones pues el número de víctimas disminuyó respecto del terremoto de 1939). No obstante, la ciudad de Valdivia post evento presentó una rápida recuperación en su crecimiento demográfico, según datos entregados por INE (1992) durante la década del 60, pues como se puede observar, se retoma el crecimiento demográfico superando la cifras pre evento, bordeando el 4\%, para luego presentar un descenso en la variación intercensal en las décadas siguientes.

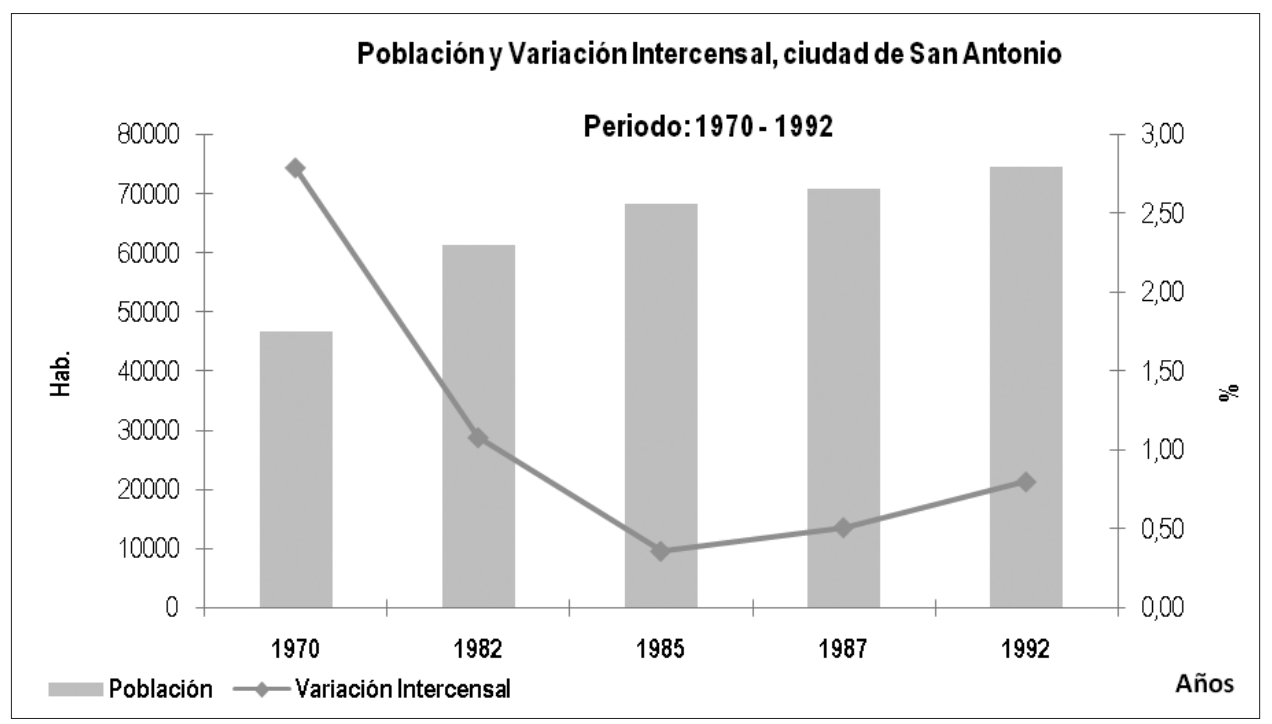

Fuente: INE. Población de los centros poblados de Chile. 1875-1992. Santiago de Chile, 1992; INE. Chile: Ciudades, Pueblos, Aldeas y Caseríos. Santiago de Chile, 2005.

16. "Los terremotos en Chile (1570-1987). Las grandes catástrofes sísmicas de nuestra historia". Documento on line: <http: //www.memoriachilena.cl/temas/index.asp?id_ut=losterremotosenla historiadechile(1570-1960)>. Fecha de consulta: 10/3/2011.

17. INE. Op. Cit., 1992. 
En 1985, y pese a la magnitud del terremoto registrado con epicentro en la ciudad de San Antonio ${ }^{18}$, solo se contabilizaron 177 muertos, cifra menor para el total de la población de ese entonces, que ascendía a 68.500 personas aproximadamen$\mathrm{te}^{19}$. Hay que señalar que la variación intercensal de la ciudad de San Antonio antes del evento presentaba un crecimiento en descenso, tal como se puede evidenciar en la gráfica; así, en el periodo 1970-1982 este fue de 2.79\% para descender a una de $0.59 \%$ para comienzos de la década del 90 . Sin embargo, un elemento que se debe tener en consideración para el análisis es el profundo impacto que tuvieron en la década de los ochenta las transformaciones en las bases económicas (crisis del 82) que afectaron profundamente la actividad portuaria de la ciudad generando una migración de población ${ }^{20}$ que no presentaba relación alguna con el fenómeno sísmico propiamente tal. Pese a ello, se observa un discreto incremento de la población que rompe la tendencia. Sin embargo, pareciera que la actividad portuaria es la que merece una exploración más en profundidad respecto del comportamiento demográfico en la zona.

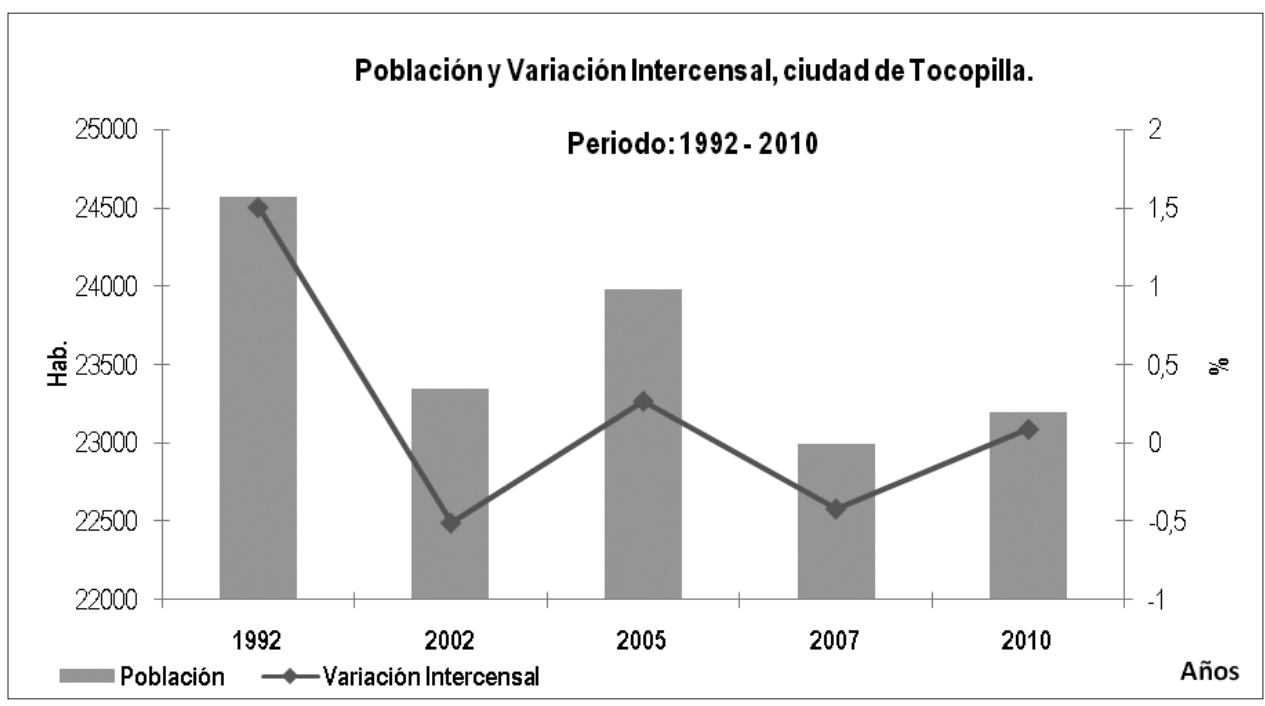

Fuente: INE. Población de los centros poblados de Chile. 1875 - 1992. Santiago de Chile, 1992; INE. Chile: Ciudades, Pueblos, Aldeas y Caseríos. Santiago de Chile, 2005.

18. <www.sismo24.cl>. Documento on line: "Listado de terremotos por ciudades". Fecha de consulta: $10 / 03 / 11$.

19. INE. Op. Cit., 1992.

20. Daher, A. "Regiones-commodities: Crisis y contagio en Chile". EURE, Santiago, v. 29, n.86, mayo 2003. 
El caso de la ciudad de Tocopilla es de difícil seguimiento en los posibles efectos demográficos de la catástrofe, pues desde antes ya su comportamiento era errático. La ciudad de Tocopilla no muestra una tendencia demográfica clara en los últimos 20 años analizados previos a la catástrofe, por lo que aventurar hipótesis sobre el efecto de la catástrofe es difícil. Pese a ello, se trata de uno de los casos que la prensa señala como ejemplo de incrementos en los nacimientos post terremoto, sin dar cifras al respecto.

\section{COMENTARIOS FINALES}

En la mayoría de los casos visualizados, la tendencia de las tasas de variación intercensal se rompe abruptamente hacia un incremento notorio con posterioridad a una catástrofe. Lo anterior es importante de tener en consideración pues pese a que en el caso del terremoto de 1939 la magnitud de la catástrofe fue de tales proporciones en términos de víctimas y de procesos migratorios, las tasas de variación intercensal aumentan de un modo interesante. Sin embargo, también es preciso ser cuidadosos en el análisis. Estas lecturas preliminares permiten solo formular algunas hipótesis sobre los procesos. Para un análisis de mayor profundidad sobre la materia, la necesidad de datos más específicos y locales resulta imprescindible. Lo más relevante sin duda, es entender que los factores que inciden en las tendencias demográficas deben abordarse aceptando y asumiendo la complejidad que subyace en estos procesos, en donde las migraciones sin lugar a dudas adquieren un peso relevante. Asimismo, el trabajo con las tasas de natalidad, que es el dato importante para una lectura más acotada y precisa sobre el fenómeno del baby boom, debe rescatar lo relativo a nacimientos registrados por localidad, que sería la única forma de probar las hipótesis referidas al fenómeno sociológico y psicológico que se vincula a las catástrofes.

Desde acá, desde una mirada de carácter más general de la tendencia demográfica, solo es posible asistir a señalar que la hipótesis de que el fenómeno del baby boom se generaría post catástrofe, parece válida y pertinente de explorar en profundidad.

Finalmente, las reflexiones que se motivan a partir de esta condición telúrica de nuestro país, permiten visualizar una serie de elementos que van más allá de las relaciones causales que se pueden establecer entre los fenómenos observados. Si bien es notorio el quiebre de tendencia demográfica con posterioridad a un evento catastrófico, también es cierto que hay una serie de elementos de mayor complejidad que están interactuando. 


\section{REFERENCIAS BIBLIOGRÁFICAS}

"200.000 nacionales destino Argentina como ayuda a damnificados del terremoto". El Sur. Concepción, Chile, 7 de febrero, 1939.

"24 enero 1939 terremoto Chillán". [En línea] <www.sismo24.cl> Fecha de consulta: 9/3/2011.

"6.026 personas evacuaron Concepción controladas por la Gobernación Marítima de Talcahuano". El Sur. Concepción, Chile, 7 de febrero, 1939.

"Alza de embarazos en la zona afectada desata un 'baby boom' en los próximos meses". El Mercurio. Santiago de Chile, 20 nov., 2010. Cuerpo C, p. 10.

Braudel, F. "Chili, cette folie géographique". Annales. Économies, Sociétés, Civilisations. 3 (4): 443-446, 1948.

"Chillán, Cauquenes, Parral, San Carlos y Linares serán evacuadas". El Mercurio. Santiago de Chile, 27 de enero, 1939, p. 1.

"Convoyes especiales condujeron a Santiago heridos y refugiados". El Mercurio. Santiago de Chile, 30 de enero, 1939. Segundo Cuerpo, p. 9.

Daher, A. "Regiones-commodities. Crisis y contagio en Chile". EURE, 29(86): 2003.

"Listado de terremotos por ciudades". [En línea] <www.sismo24.cl> Fecha de consulta: 10/3/2011.

"Los terremotos en Chile (1570 - 1987). Las grandes catástrofes sísmicas de nuestra historia. [En línea] <http: //www.memoriachilena.cl/temas/index.asp?id_ut=losterremotose nlahistoriadechile(1570-1960)>. Fecha de consulta: 10/3/2011.

Ministerio del Interior. Balance de Reconstrucción a un año del 27/F. [En línea] <http: // issuu.com/minterior/docs/divest_10207_estudios_especiales_balance_a_1_a_o_d?mo de=a_p\&wmode=1>. Fecha de consulta: 7/3/2011.

Población de los centros poblados de Chile. 1875-1992. Santiago de Chile, Instituto Nacional de Estadísticas, 1992.

Russell, C. The baby boom: Americans born 1946 to 1964. New Strategist Publications, NY, 2009.

Sismos importantes y/o destructivos (1570 - a la fecha). [en línea] Universidad de Chile. Departamento de Geofísica. Servicio Sismológico. <http: //ssn.dgf.uchile.cl/seismo. html >. Fecha de consulta: 8/3/2011.

Subercaseaux, B. Chile o una loca geografía. Ediciones Ercilla, 1946. 